\title{
Terrorism and Crime Related Weblog Social Network: Link, Content Analysis and Information Visualization
}

\author{
Christopher C. Yang and Tobun D. Ng
}

\begin{abstract}
A Weblog is a Web site where entries are made in diary style, maintained by its sole author - a blogger, and displayed in a reverse chronological order. Due to the freedom and convenience of publishing in Weblogs, this form of media provides an ideal environment as a propaganda platform for terrorist groups to promote their ideologies and as an operation platform for organizing crimes. In this work, we present a framework to analyze and visualize Weblog social network embedded beneath relevant Weblogs gathered through topic-specific exploration. Link analysis uses the relationships between bloggers to construct the Weblog social network. Content analysis associates similar blog messages to unveil implicit relationships found in the semantics to further improve the Weblog social network analysis. Users can use different interactive information visualization techniques to explore various aspects of the underlying social network at different levels of abstraction. With the capability of analyzing and visualizing Weblog social networks in terrorist and crime related matters, intelligence agencies and law enforcement will be able to have an additional tools and means to ensure the national security
\end{abstract}

\section{INTRODUCTION}

THE ease of publishing in the Web together with the individuals' willingness and desire to share their thoughts has been the catalyst for the fast and continuously growing of Weblogs. As of mid-December 2006, a Weblog search engine, Technorati, was tracking about 62.5 million Weblogs, with 175,000 newly created weblogs everyday and over 1.6 million posts updating to existing Weblogs per day [9]. Furthermore, the social impact of Weblogs comes from not only the large number of individual Weblogs but also the underlying Weblog social network formed by the interconnection and interaction between bloggers. Thus, Weblogs provides a very rich source for mining the thoughts of individuals and their social network as a whole. Contrary to the explicit relationships between members in forums, some of the important relationships are embedded inside the semantics of bloggers' messages. This posts a challenge in analyzing Weblog social networks.

Weblogs are considered as online diaries authored by individual users with the following characteristics: 1) ordered with reverse chronological sequences with time stamps, 2) associated with a profile of the authors with information including name, geographic location, date of birth, interests, etc., 3) maintained and published with the help of a popular blog authoring tool, and 4) quirky, highly personal, read by repeat visitors, interwoven into a network of a tight-knit but active communities [4,5].

C. C. Yang and T. D. Ng are with the Department of Systems Engineering and Engineering Management at the Chinese University of Hong Kong (e-mail: yang@se.cuhk.edu.hk).
Weblogs have become a prevailing type of media on the Internet. Bloggers are free to express their opinions and feelings to hundreds of millions of Internet users around the world without control of any corporate media. Rosenbloom [8] identified a number of ways how bloggers use Weblogs: 1) share any personal detail that comes to mind, generally to a small circle of family members and friends, 2) amplify the voices of politicians, pundits, scholars, researchers, and journalists - public-spirited blogs, 3) establishing and maintaining online communication in any topic interests, 4) promoting new products or mounting a public defense in the wake of bad news about faulty products or falling stock prices by businesses, 5) discussing entertainment subjects such as celebrity gossip. In addition, Nardi et al. [6] found that bloggers are motivated due to several reasons: documenting one's life, providing commentary and opinions, expressing deeply felt emotions, articulating ideas through writing, and forming and maintaining community forums.

Due to the freedom and convenience of publishing in Weblogs, this form of media provides an ideal environment as a propaganda platform for extremist or terrorist groups to promote their ideologies. Criminals may also make use of the virtual environment to organize crimes such as money laundering and drugs trafficking without being easily identified. As a result, it is important to understand the social network of the bloggers in order to assess the risks that may threaten the national security.

Although the Weblogs are so popular, the blog searching is not as advanced as Web searching. The performance of Weblog search such as Google Blog Search is not as satisfactory as those obtained from searching Web pages. A Weblog contains hundreds of messages and the messages are updated frequently. Indexing such Weblog is not as easy as indexing a Web page because a Weblog may cover many different topics and therefore keyword matching may not work well in Weblog. Hyperlink analysis such as PageRank that is used in Google does not work on Weblog because bloggers usually do not make hyperlinks to other Weblog while they are writing their own message. Most bloggers find blogs through other blogs they are reading or through inclusion of the blog link in an instant message profile or Web page [6].

In order to harvest relevant social relationships and shared ideas from the enormous Weblog space, we propose to develop a crawler to extract the Weblog sub-community on specific topics. Section II provides insight about the implicit and explicit relationships in Weblog social networks. Section III describes a framework for supporting query-dependent exploration and analysis in relevant Weblog sub-communities. Section IV discusses the use of link analysis and content analysis to support the searching and information analysis of the community with a specific topic. 
In Section IV, interactive information visualization techniques will also be developed to support the exploration of the social network and extraction of information. Section VI discusses some potential applications and users who will be benefited from the proposed work.

\section{WEBLOG SOCIAL NETWORK}

Web search and ranking algorithms such as PageRank and HITS are conducting hyperlink analysis on the Web social network with nodes and arcs corresponding to Web pages and hyperlinks [1]. Based on the in-links and out-links of nodes, the PageRank score or the authoritative and hub scores of each Web page are computed. In the University of Arizona's Dark Web project [13], social networks of the US domestic extremists and hate groups are constructed. The nodes and arcs correspond to the extremists and hate groups' Web sites and their hyperlinks. Link and content analyses are then conducted to investigate the relationships among the extremist and hate groups. Yang et al. [12] has also investigated the terrorist social network where the nodes and arcs correspond to terrorists and their relationships, such as acquaintance, relatives, nuclear family member, religious leader, etc.

In Weblog social network, nodes correspond to blog pages where each blogger has his or her blog page. For example, blogger "abc" has a blog page with URL "http://www.xanga. com/abc". However, most bloggers usually do not have hyperlinks to other blog pages in their messages because most bloggers are only sharing their own thinking, feeling or amplifying their opinions. The arcs on a Weblog social network correspond to the interaction between the bloggers, which can be identified through the information on the profile or the messages. We identify four types of blogger interactions based on the Xanga blog site: 1) subscription, 2) friends, 3) comments, and 4) recommendation. The first two types of interactions, subscription and friends, are the relationships that bloggers declare based on the general interest on each other. In every blog page, there is a function for other bloggers to subscribe to his blog page. The subscriber will be alerted when the blogger, who he has subscribed to, posts a new message. When a blogger $A$ subscribes to the blog page of blogger $B$, there is a directed arc from $A$ to $B$ in the Weblog social network based on subscription, BSN-S. A blogger may also claim other bloggers as his friends. When a blogger $A$ claims a blogger $B$ as his friend, there is a directed arc from $A$ to $B$ in the Weblog social network based on friends, $B S N-F$. The last two types of interaction are developed based on the messages. A blogger may make a comment on another blogger's message. If a blogger $A$ make a comment on a blogger $B$ 's message, a directed arc will appear from $A$ to $B$ in the Weblog social network based on comments, $B S N-C$. A blogger may read another blogger's message and make a recommendation to other bloggers although he may not make any comments on the message. When a blogger $A$ makes a recommendation of the message posted by blogger $B$, there is a directed arc from $A$ to $B$ in the Weblog social network based on recommendation, $B S N-R$. The arcs on $B S N-S, B S N-F$,
$B S N-C$, and $B S N-R$, imply different strength of association between the corresponding bloggers. When a blogger subscribes to another blogger, it means that he is interested in the messages posted in general but not necessary on every single message in the blog page. On the other hand, when a blogger makes comments on a specific message, it means that he is interested in the specific content in the message; however, he may not read any other messages on the blog page. Social network analysis can be conducted on each $B S N$ s or conducted on the overall $B S N$ by superimposing $B S N-S, B S N-F, B S N-C$, and BSN-R.

\section{QUERY-DEPENDENT SCHEME VS. QUERY-INDEPENDEN SCHEME}

The hyperlink analysis in Web searching and ranking can be classified into two schemes: query-dependent scheme and query-independent scheme [1]. In the query-independent scheme, a spider continues to crawl new Web pages through the hyperlinks and constructed a network that includes all Web pages. Scores will be assigned to Web pages once they are in regardless of the queries. PageRank that is used in Google search engine is a typical example [3]. In the query-dependent scheme, a query-specific graph (or know as neighborhood graph) is constructed. A query is first submitted to a search engine to obtain the top $\mathrm{N}$ documents to form a seed set. The Web pages that have in-links or out-links from the seed set will be crawled to form the neighborhood. Scores on each Web page in the neighborhood graph will then be computed. HITS is a well-know query-dependent ranking algorithm.

In this work, we focus on the interactions between the bloggers on specific topics, especially on the terrorism and crime related topics. As a result, we adopt the query-dependent scheme on our Weblog social network analysis. We submit a query such as "London liquid bomb" and "Sadam Hussein" to Weblog search engines (e.g. Xanga.com and Google Blog Search) to build the seed set. Based on the four types of relationships, subscription, friends, comments, and recommendations as described in Section II, we develop four types of BSNs. Link and content analyses will then be conducted to extract useful patterns. Users may also make use of the information visualization techniques to explore the social network. Other applications such as classifications, theme and structure evolution, sub-graph extraction, sentiment analysis and threat analysis are developed on top of link and content analyses as well as information visualization.

We create a framework, as shown in Figure 1, to bring various research and technical components together for analyzing and understanding terrorism and crime related Weblog social networks. This framework has four main components: Weblog discovery and collection, Weblog analysis, user interface and interactive information visualization, as well as applications. The Weblog discovery keeps track of existing Weblogs and monitoring any emerging Weblogs from known blog sites or web communities for forums and chat rooms. The crawler is then based on Weblog seeds and user query to collect relevant 


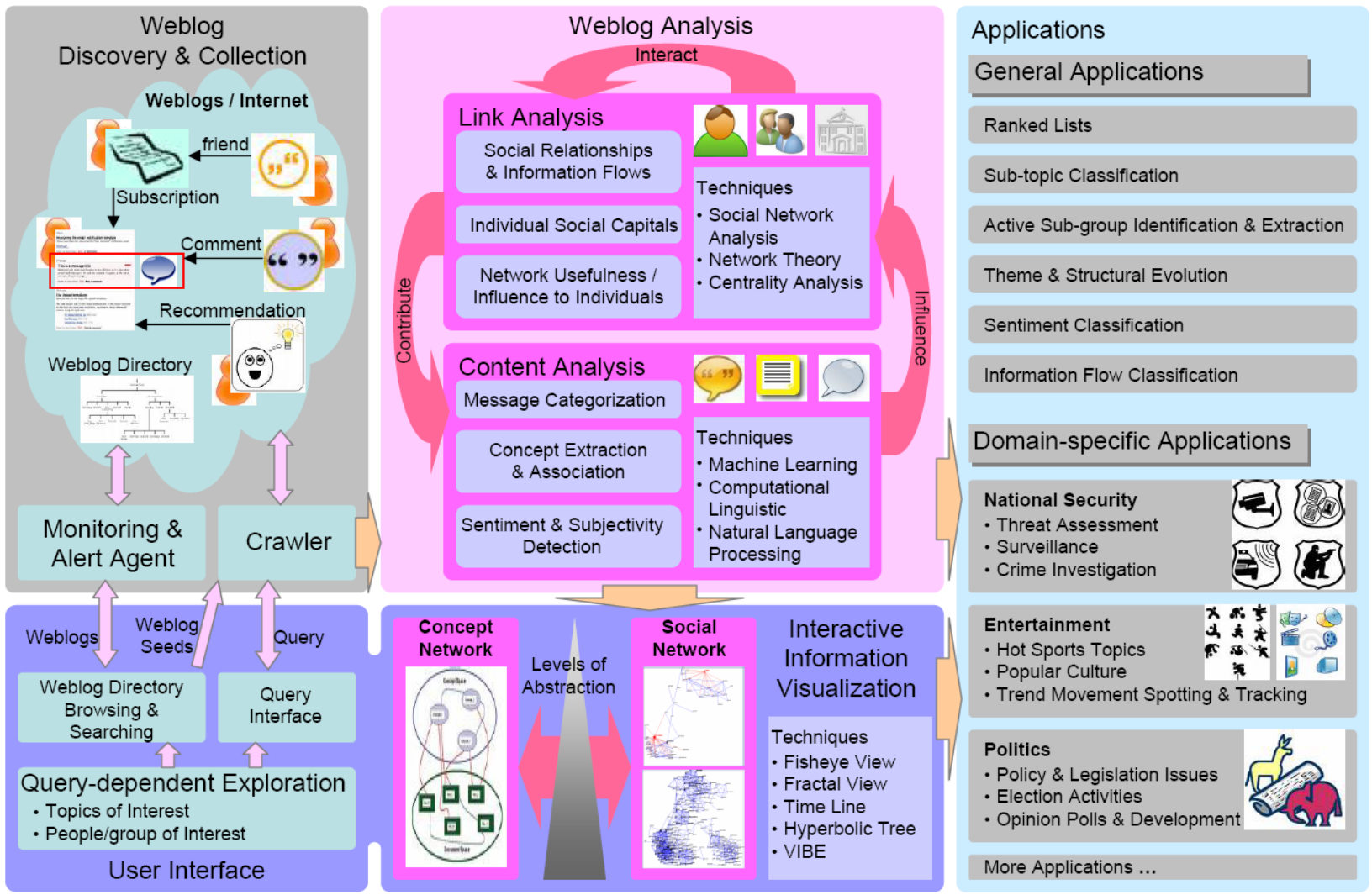

Figure 1. Framework of the proposed Terrorism and Crime Related Weblog Social Network project

Weblogs for further analysis. The Weblog analysis is not only to unveil the interactive relationship between individuals, groups, or organizations but also to mine the content and context of the messages and information flow. The details of the Weblog analysis will be discussed in Section IV. The user interface allows users to drive query-dependent exploration with specific query and selected set of Weblog seeds choosing from the results of Weblog search engines. Once Weblogs have been collected and analyzed, users will be able to use the interactive information visualization techniques to investigate various aspects of the underlying social networks. Section V will discuss more about the interactive information visualization. Section VI will discuss the development of different applications based on the results of Weblog analysis and the techniques in information visualization.

\section{BLOG ANALYSIS: LINK ANALYSIS AND CONTENT ANALYSIS}

The mass of a Weblog collection builds upon interactive relationships between bloggers and information flows within the network. The essence of the blog analysis is to provide some understanding of the synergetic affects during the evolvement of blogger's interactions, content contributions, and different degrees of influence from expressed thoughts. The link analysis focuses on understanding the people aspect of the underlying social network while the content analysis aims to comprehend the content and context in the Weblog messages and the information flows within the network.
Social network analysis views social relationships in terms of nodes and links. Nodes represent the bloggers, i.e., individuals, groups, or organizations, within the networks while links represent relationships between the bloggers. The types of relationship existing in the Weblogs are subscriptions, friends, comments, and recommendations, as described previously in Section II. The quantity, directions, and types of links between nodes can be used to analyze the social capitals of individual bloggers in a social network. The social capital is the advantage created or gained by an individual's position in a structure of relationships [7]. It explains how some people or groups gain more success or influencing power in a social network through their superior connections to other individuals. The social capital can be quantified by the measures of centrality and prestige of each node in the social network. Centrality of a node can be measured by degree centrality, closeness centrality, and betweenness centrality. The degree centrality measures the number of links to other nodes in the network. The closeness centrality measures the distance from a node to all other nodes; that is, the closeness is the inverse of the sum of the shortest distances between each node and every other node in the network. The betweenness centrality measures the control of a node over other pairs of nodes in a network. The prestige measures the in-degree of a node.

Other than analyzing the social capitals of individual bloggers in the social networks, it is important to understand networks' usefulness or influence to their members. Relatively smaller and tighter networks can be less useful or influential to their members than networks with plenty of 
loose or weak connections to individuals outside the main network. More loose networks, with many weak ties and social connections, are more likely to introduce new ideas and opportunities to their members than closed networks with many redundant ties [2].

Some of the weak ties are indeed difficult to recognize in the current setting of Weblogs. The recommendation relationships, BSN-R, do not have any structural or syntactic support in describing them in Weblogs. These relationships are embedded in the semantics of bloggers' messages. Content analysis can be employed to extract the content with context for revealing such implicit relationships. In addition, it is possible to have two or more bloggers who have never been interacted to express similar thoughts on similar topics. Text classification will be able to group similar ideas and provide additional linkages between different bloggers.

A blogger is free to write his or her ideas in any length or style. That is, a blog message may contain more than a topic or idea. It will be beneficial to segment a message into a set of logical units for content analysis. Another difficulty in analyzing blog text is that messages may not be written with correct grammar or in correct sentence structure. This will post technical difficulties in using natural language processing techniques to analyze the content. Some other computational linguistic techniques like bi-grams or tri-grams may be useful for the task. Because a blog message highly reflects a blogger's emotional state, it will be fruitful to analyze the sentiment and subjectivity of the message [10].

\section{INTERACTIVE INFORMATION VISUALIZATION}

Interactive information visualization allows users to explore the Weblog social network by selecting the focuses of interests and adjusting the parameters of the visualization tools such as fisheye view and fractal view [11]. Fisheye view enlarges the region of interest and diminishes other regions while maintaining the global structure. Fractal view abstracts the complex structure by filtering the nodes and arcs that are less relevant to the focus of interest in order to reduce the visual loading of users. By selecting the nodes in the Weblog social networks as focus points, users can extract the active sub-groups, identify the important actors and their roles, and discover interaction patterns of the bloggers.

Through the visualization tools, users may identify the theme in the discussion of each active sub-group and the evolution of the theme within the community [12]. To further understand their specific content, users can clicks on the nodes to read the messages they post and how they respond to each other.

\section{APPLICATIONS}

The capability of analyzing and viewing Weblog social networks from various aspects at different levels of abstraction is the foundation to develop applications to meet different users' needs. From the perspective of digital library, the general public will be able to have informative and insightful access to ever-evolving Weblogs or Weblog communities through some general applications like ranked lists of top bloggers, active sub-group identification and extraction, theme and structural evolution of Weblog social networks, as well as classification of sub-topics, sentiment, and information.

Gathering intelligence is a vital step to prevent and fight against terrorism and crime related matters. Applications in the domain of national security will enable intelligence agencies and law enforcement to discover potential threats and assess their threat levels through the monitoring of the content development and the activeness of the interaction in Weblog social networks. In addition, information surveillance and crime investigation can be performed with the ability to analyze Weblog social networks. Likewise, other domains such as entertainment and politics can be benefited from Weblog social network analysis because of the tremendous potential to tap directly into millions of individual's minds.

\section{CONCLUSION}

In this work, we have introduced a framework to analyze Weblog social networks gathered by a query-dependent exploration scheme. The Weblog social network analysis utilizes the content analysis to extract semantics and topics, and then associate bloggers who have never been interacted. Furthermore, the link analysis will use all explicit and implicit relationships between Weblogs to create their Weblog social network. Interactive information visualization techniques like fisheye view have been introduced for users to explore various aspects at different levels of abstraction about the underlying social network. We have also described the applications developed from the link and content analyses as well as visualization techniques can greatly benefit the operations in national security and even the general public to gain insight into any Weblog social network.

\section{REFERENCES}

[1]. G. W. Flake, C. L. Giles, and F. M. Coetzee, "Self-Organizing and Identification of Web Communities,” IEEE Computer, March, 2002, 66-71.

[2]. M. Granovetter, “The Strength of Weak Ties," American Journal of Sociology, 78(6), May 1973, pp. 1360-1380.

[3]. M. R. Henzinger, "Hyperlink Analysis for the Web," IEEE Internet Computing, January/February, 2001, pp.45-50.

[4]. R. Kumar, J. Novak, P. Raghavan, and A. Tomkins, "Structure and Evolution of Blogspace,” Communications of the ACM, 47(12), December, 2004, pp.35-39.

[5]. Q. Mei, C. Liu, H. Su, C. Zhai, “A Probabilistic Approach to Spatiotemporal Theme Pattern Minning on Weblogs," Proceedings of the International World Wide Web Conference (WWW 2006), May 23-26, Edinburgh, Scotland, 2006.

[6]. B. A. Nardi, D. J. Schiano, M. Gumbrecht, and L. Swartz, "Why We Blog," Communications of the ACM, 47(12), December, 2004, pp.41-46.

[7]. A. Portes, Social Capital: Its Origins and Applications in Modern Sociology, 1998.

[8]. A. Rosenbloom, “The Blogoshpere," Communications of the ACM, 47(12), December, 2004, pp.31-33.

[9]. Technorati, Inc., “Technorati: About Us," http://technorati.com/about/, 2006.

[10]. J. Wiebe and R. Mihalcea, "Word Sense and Subjectivity," COLING-ACL-2006, Sydney, July, 2006, pp. 1065-1072.

[11]. C. C. Yang, N. Liu, and M. Sageman, "Analyzing the Terrorist Social Networks with Visualization Tools," Proceedings of the IEEE International Conference on Intelligence and Security Informatics, San Diego, CA, US, May 23 - 24, 2006.

[12]. C. C. Yang, X. Shi, and C. Wei, "Tracing the Event Evolution of Terror Attacks from On-line News," Proceedings of the IEEE International Conference on Intelligence and Security Informatics, San Diego, CA, US, May 23 - 24, 2006.

[13]. Y. Zhou, E. Reid, J. Qin, H. Chen, and G. Lai, "US Domestic Extremist Groups on the Web: Link and Content Analysis," IEEE Intelligent Systems, September/October, 2005, pp.44-51. 